\title{
Unmanned Leg-Wheel Vehicle Design and of Vertical Step Passability Performance Analysis
}

\author{
Ruotian Wang, Huaying Li \\ Department of Mechanical Engineering, Academy of Armored Force Engineering, Beijing 100072, China;
}

Yunhai Wang, Zhixiong Wang

Jiuquan Satellite Launch Center, Jiuquan 732750, China

\begin{abstract}
In order to achieve high mobility for country crossing and the ability of climbing vertical obstacles, a new locomotion mechanism that combines legs and wheels is proposed, and a prototype mobile robot that adopts the mechanism is introduced, actions for obstacle climbing is designed. Focused on forces and power requirements on leg-wheel mechanism in the process of obstacle climbing, calculated the maximum of output torque during motor drive legs rotation, established a model of unmanned ground vehicle through the multibody dynamics modeling software ADAMS, simulated and analyzed the process of obstacle climbing. Result show that the proposed structure meet the demand of $220 \mathrm{~mm}$ high platform climbing with the maximum rotating torque $4.99 \mathrm{~N} \cdot \mathrm{m}$.
\end{abstract}

KEYWORD: unmanned ground vehicle, obstacle climbing, leg-wheel structure.

\section{INTRODUCTION}

A. Ground Wheeled/Legged vehicle

Ground wheeled vehicles with simple mechanical structure, move effectively and easy to control. However, their simple and robust design does not provide sufficient versatility and adaptability for many realworld terrain conditions which limit its application for unstructured environment. Legged structure vehicles are easy to pass the uneven ground, but with a low driving efficiency and complex kinematic control method. (Lu D, 2013) Leg-wheel ground vehicles integrated the advantages of wheeled and legged structure are of high mobility and resistance, widely used in planetary exploration, disaster field rescue, military reconnaissance missions.

B. Vertical Step Passability for UGV (Unmanned Ground Vehicle)

As a matter of fact, unmanned ground leg-wheel vehicles for urban reconnaissance offer an efficient and often rapid method of ground traversal, especially in conditions where the terrain is flat, which make passability of complex environment the most important criterion.( J. A. Smith, 2006) Up to the present, many leg-wheel hybrid type vehicles have been developed. For example, the Roller-Walker, a vehicle based on roller-skating technique, with special foot mechanism which changes to the sole for walking mode and passive wheels for wheel mode. ( S. Hirose, 1996).The spider like quadruped Air Hopper, with a jumping height of $850 \mathrm{~mm}$ vertically, and landed on the ground softly. The wheeled-legged hybrid robot
Wheeleg has two pneumatically actuated front legs, each with three degrees of freedom and two rear wheels independently actuated.

In this paper, for urban environment reconnaissance mission, we proposed an unmanned leg-wheel vehicle, four legged structures rotate in omni-direction, set of wheels mounted on the hub motors at the end of 4 legs for driving the vehicle. To achieve reasonable design and meet the needs of vertical step passability, a multi-step passing method and studied force characteristics with the analysis of dynamic parameters; simulated the torque in rotating arm to obtain the maximum torque in demand.

\section{STRUCTURE AND VERTICAL STEP CLIMBING DESIGN}

As for urban reconnaissance, the vehicle should be small, light weight, high passablity of unstructured environment. Design specifications are as follows.

Table 1 Design Specifications of Leg-Wheel Vehicle

\begin{tabular}{ccccc}
\hline $\begin{array}{c}\text { Struc- } \\
\text { ture }\end{array}$ & Weight/kg & Load/kg & $\begin{array}{c}\text { Minimum } \\
\text { Size } \\
\left(\mathrm{a}^{*} \mathrm{~b}^{*} \mathrm{~h}\right) / \mathrm{mm}\end{array}$ & $\begin{array}{c}\text { Step } \\
\text { Height } \\
/ \mathrm{mm}\end{array}$ \\
\hline $\begin{array}{c}\text { Leg- } \\
\text { Wheel }\end{array}$ & $<20$ & $>5$ & $500 * 500 * 150$ & $>200$ \\
\hline
\end{tabular}




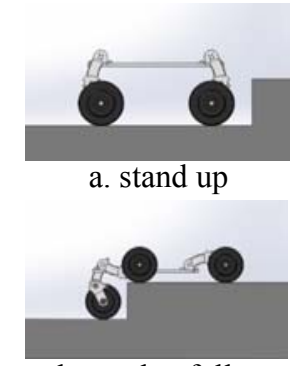

d. rear leg follow

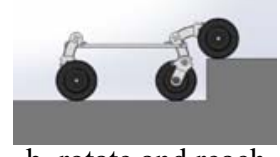

b. rotate and reach

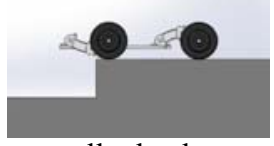

e. all wheels up

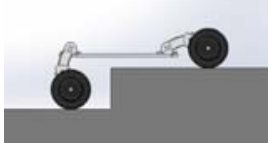

c. rotate and follow

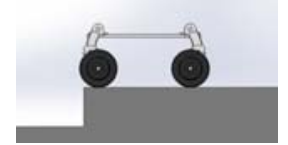

f. finished
Fig. 1 Unmanned Leg-Wheel Structure

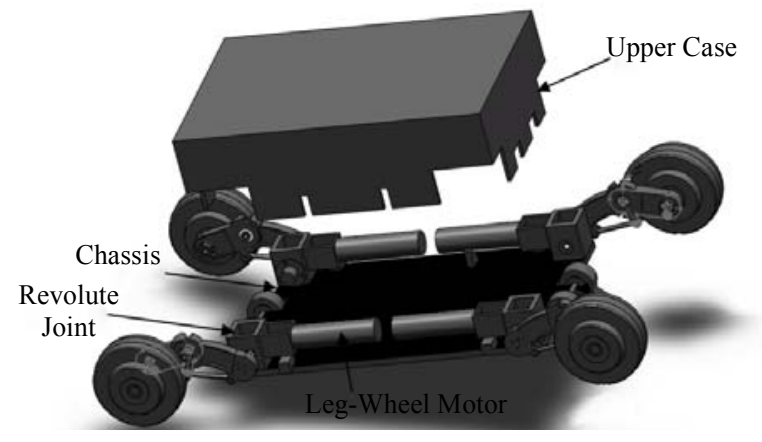

Fig. 2 Step Designed for Vertical Passability

Structure Design. To achieve above goals, a legwheel vehicle was designed as shown in FIG. 1. Flanked by four legs steering shaft, the torque from the driving motor is transmitted from the worm gear to the legs' shaft, drive leg structures around the axis in $360^{\circ}$ rotation; in leg knee structure joint with innerspring damping suspension system designed with limitation in position of $\pm 15^{\circ}$ swinging degrees; legs swing so that vehicles pose can be changed dynamically.

Vertical Step Climbing Design. In order to ensure stable movement, the lower center of gravity is better for obstacle climbing, when faced with high platform, the motor driven wheel legs to form an upright posture, giving the platform a higher pose for pre-climbing.( Hughes A, 2015) As FIG.2(a) shows. Then one of the front legs rotates $3 \pi / 2$ to reach the platform, as FIG.2(b) shows. After that, the other front leg rotate and rear wheels move forward to follow and reach the platform, showed in FIG.2(c).(d). Then all of the wheels stands on the platform and change back to the former upright posture, as shown in FIG.2(e).(f).

\section{DYNAMIC PROCESS AND POWER DEMAND ANALYSIS}

Action design based on the obstacle climbing should match the power system and motion sources. 4 legwheel driving motors provide the torque for leg rotation, processing stepwise actions to meet the entire execution. To this end, established the dynamic model of the unmanned vehicle to analyze the me- chanical characteristics of the loading limit, maximum stress as a criterion of power capability. The model can be simplified by following rules: 1) Leg swing in uniform rotation in the entire rotation process. 2) Hub motors are light weight in comparison with vehicle body so that mass can be ignored. 3) Assume the centroid of the body as the center of gravity.

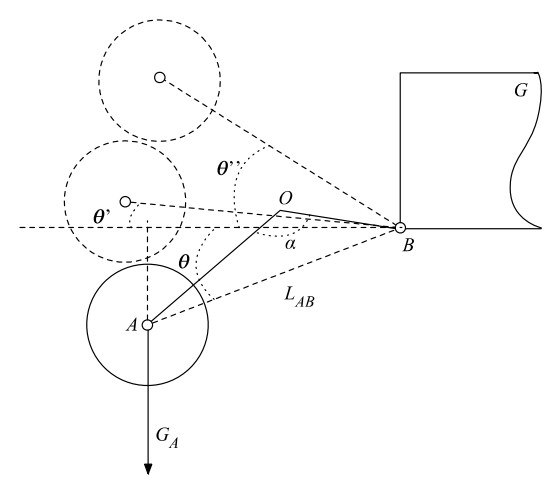

Fig. 3 Single Wheel Rotation Model

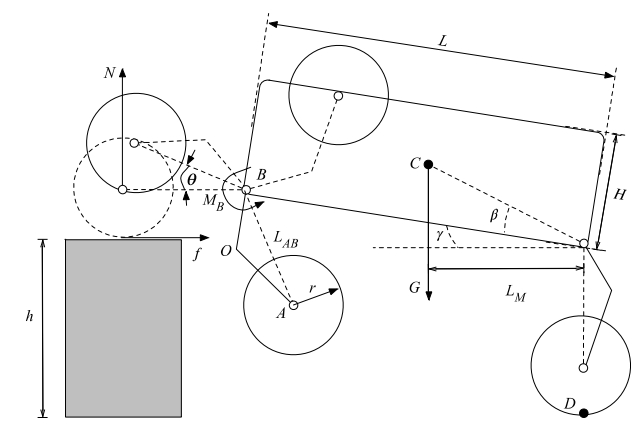

Fig. 4 Maximum Analysis of Climbing Process

Limit Force Analysis of Single Rotational Leg. Single wheel rotation model is shown in FIG.3.

Point A is hub motor center, Point B transmit the torque from rotating motor so that the legs rotate in 360 degrees. To drive the legs rotate full circle, a torque balance should accomplish as follows:

$M \mathrm{~B}=\mathrm{G}_{\mathrm{A}} \mathrm{LAB}_{\mathrm{AB}} \cos \theta$

From formula (1), result shows that when the leg is parallel with the horizontal plane, the torque of shaft $B$ reach the maximum of $3.605 \mathrm{~N} \cdot \mathrm{m}$.

Supporting Action Stress Analysis. As is shown in FIG.4, according to the aforementioned obstacle climbing designed actions, to accomplish a $220 \mathrm{~mm}$ high platform climbing, the process of the minimum torque can be derived from formula (2) which imply the necessary conditions to meet the design performance. As the front leg and centroid form cause a leverage, 2 legs share the stress for supporting the vehicle, because of the front 2 legs are symmetrical, only one side force analysis is sufficient. (A. De Luca, 1998) Therefore point $D$ can be regarded as the reference point, the entire swing process with uniform rotation satisfy the condition below: 
$N\left(\mathrm{~L}_{\mathrm{AB}} \cos \theta+\mathrm{L} \cos \gamma\right)+f \mathrm{~h}=\frac{1}{2} \mathrm{G} L_{\mathrm{M}}$

$L_{\mathrm{M}}=\frac{\sqrt{\mathrm{H}^{2}+\mathrm{L}^{2}}}{2} \cos (\gamma+\beta)$

$\gamma=\arcsin \frac{\mathrm{r}+\mathrm{h}-\mathrm{L}_{\mathrm{AB}}(1-\sin \theta)-\mathrm{r}}{\mathrm{L}}$

$\beta=\arctan \frac{\mathrm{H}}{\mathrm{L}}$

$M_{\mathrm{B}}=N \mathrm{~L}_{\mathrm{AB}} \cos \theta+f\left(\mathrm{r}-\mathrm{L}_{\mathrm{AB}} \sin \theta\right)$

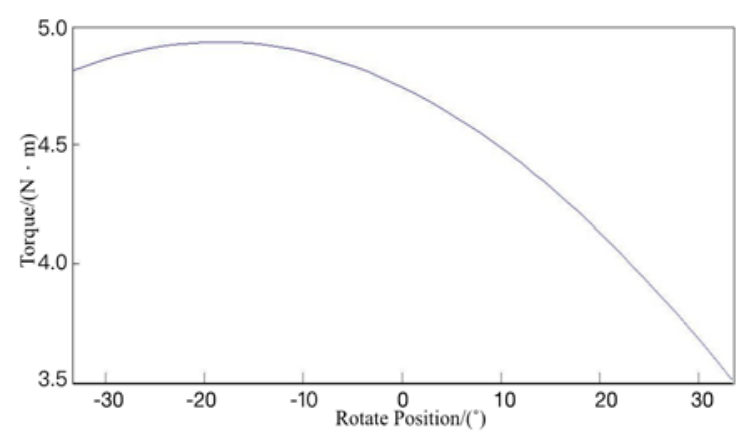

Fig. 5 Torque and Rotating Angle Relation

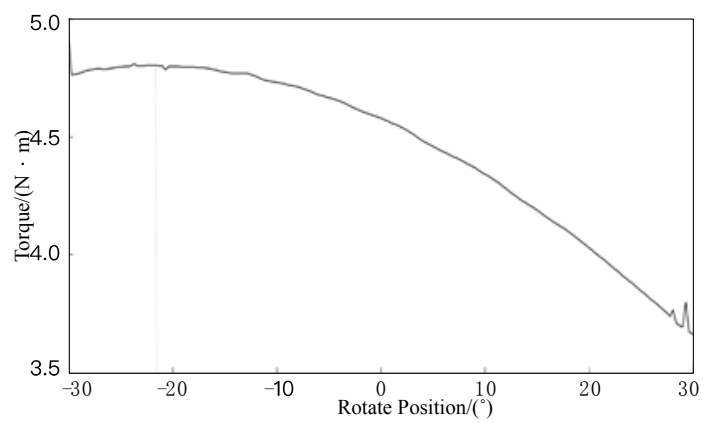

Fig. 6 Simulation Results in ADAMS

According to the aforementioned formula, using MATLAB to derive a torque value and arm position curve shown as is shown in FIG.5, it is found that when $\theta=-18.8^{\circ}$ torque from shaft $\mathrm{B}$ has a maximum value of $4.93 \mathrm{~N} \cdot \mathrm{m}$.

\section{VERIFICATION FOR SIMULATED MODELS}

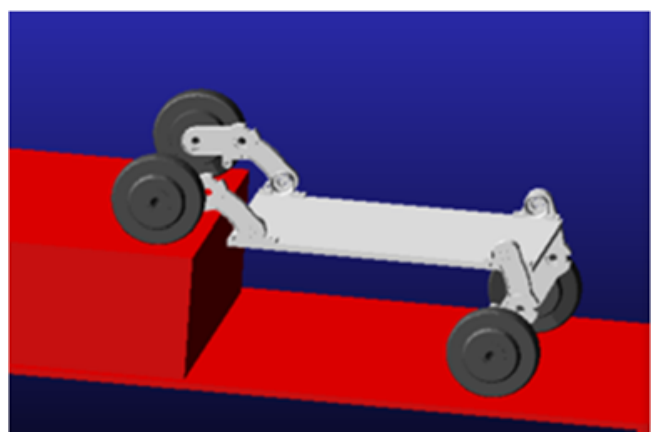

Fig. 7 Initial State

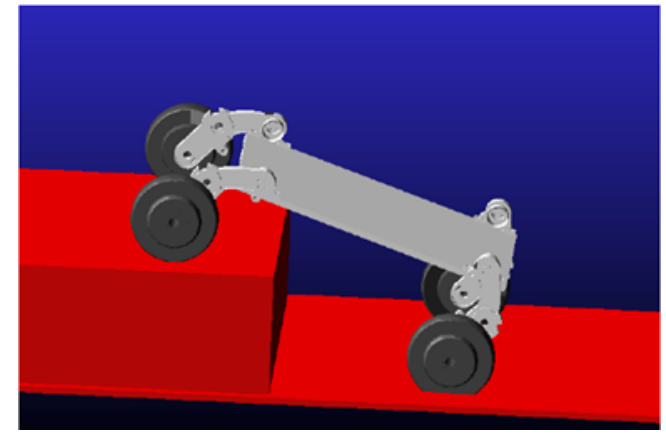

Fig. 8 End Position

Using ADAMS to analyze the process of leg rotation driven by the motors, from initial state of standing horizontal to end position that centroid reach the height for passing the platform. Using ADAMS/Measure module to measure the torque provided by the leg motors, then obtained the relation between rotate position and torque from ADAMS/ PostProcessor module as is shown in Fig. 6. Torque curve shows that the motor torque reaches the maximum value at -16 degree, which has a difference between the theoretical value of $2.3 \%$. Possible reasons for the error could be: on one hand, there is a certain flexibility in contact constraints simulation model, but the theoretical model is based on the pure rigid model; on the other hand, mass of the body is considered to be in the centroid of the vehicle, which may not reflect the reality of mass distribution.

\section{SUMMARY}

Depending on the application background, a legwheel mechanism action was designed. Based on the deformable structure a set of obstacle climbing actions was introduced. By examining the obstacle climbing process, determines the output torque of the motor drive system with the variation of the angle of rotation and limit state, which has come to be not less than $220 \mathrm{~mm}$ vertical step onto the high platform. Interactive design prove the calculation of demand torque meet the dynamic simulation model, which lay a foundation for dynamic system design.

\section{REFERENCES}

A. De Luca, G. Oriolo and C. Samson, Robot Motion Planning and Control. Springer, London (1998).

Eric Rohmer, Giulio Reina, Kazuya Yoshida. Dynamic Simulation-Based Action Planner for a Reconfigurable Hybrid LegWheel Planetary Exploration Rover(J). Advanced Robotics, 2010, 24(8-9):1219-1238.

Hughes A, Hughes A. Electric Motors and Drives(M). Elsevier LTD, Oxford, 2005.

J. A. Smith, I. Sharf, and M. Trentini. "PAW: a Hybrid WheeledLeg Robot." In Proceedings of the 2006 International Conference on Robotics and Automation, pp. 4043 - 4048, Orlando, FL, USA, May 2006. 
Lu D, Dong E, Liu C, et al. Mechanical system and stable gait transformation of a leg-wheel hybrid transformable $\operatorname{robot}(\mathrm{C}) / /$ Advanced Intelligent Mechatronics (AIM), 2013 IEEE/ ASME International Conference on. IEEE, 2013:530-535.

S. Hirose and H. Takeuchi, "Study on roller-walk (basic characteristics and its control)," in Robotics and Automation, 1996. Proceedings., 1996 IEEE International Conference on, 1996, pp. 3265-3270.

T. Tanaka and S. Hirose, "Development of leg-wheel hybrid quadruped "AirHopper" design of powerful light-weight leg with wheel," in Intelligent Robots and Systems, 2008. IROS 2008. IEEE/RSJ International Conference on, 2008, pp. 38903895 . 